\title{
Severe Pulmonary Hypertension in Adult Pulmonary Langerhans Cell Histiocytosis: The Effect of Sildenafil as a Bridge to Lung Transplantation
}

\author{
Takayuki Yoshida $^{1}$, Satoshi Konno ${ }^{1}$, Ichizo Tsujino ${ }^{1}$, Takahiro Sato ${ }^{1}$, Hiroshi Ohira ${ }^{1}$, \\ Fengshi Chen ${ }^{2}$, Hiroshi Date ${ }^{2}$, Akihiro Ishizu ${ }^{3}$, Hironori Haga ${ }^{4}$, \\ Mishie Tanino ${ }^{5}$ and Masaharu Nishimura ${ }^{1}$
}

\begin{abstract}
Severe pulmonary hypertension $(\mathrm{PH})$ often develops in patients with pulmonary Langerhans cell histiocytosis $(\mathrm{PLCH})$. Supplemental oxygen treatment is often used, whereas pulmonary arterial hypertension-specific vasodilators are generally considered hazardous because of the possible development of pulmonary edema and deterioration of hypoxia. In the present report, we herein describe a PLCH patient with severe PH in whom sildenafil, a phosphodiesterase 5 (PDE5) inhibitor, substantially improved the pulmonary hemodynamics before lung transplantation. An immunohistochemical study of the resected lung revealed positive staining for PDE5 on the diseased pulmonary arteries. These observations suggest that sildenafil can be a promising therapeutic option for PH in patients with PLCH.
\end{abstract}

Key words: pulmonary hypertension, pulmonary Langerhans cell histiocytosis, sildenafil

(Intern Med 53: 1985-1990, 2014)

(DOI: 10.2169/internalmedicine.53.1772)

\section{Introduction}

Pulmonary Langerhans cell histiocytosis (PLCH) is a smoking-related disease of an undetermined etiology that predominantly affects young adults (1-4). The disease can regress spontaneously after smoking cessation, but it sometimes progresses to chronic respiratory insufficiency (5-7).

Pulmonary hypertension $(\mathrm{PH})$ is a common complication that develops during the course of advanced PLCH. Supplemental oxygen, diuretics, and ultimately lung transplantation have been the conventional options for treating PLCHassociated PH (PH-PLCH). Pulmonary arterial hypertension (PAH)-specific vasodilators have long been considered hazardous because of the risk of pulmonary edema and hypoxia in patients with obstructions in the pulmonary veins. However, recent studies have described that PH-PLCH patients treated with PAH-specific drugs did not have hypoxia or exacerbating pulmonary edema $(8,9)$.

We herein describe a patient with PH-PLCH who was treated with sildenafil, a phosphodiesterase type 5 (PDE5) inhibitor used as a bridge to lung transplantation. This significantly improved the patient's hemodynamics without affecting oxygenation or causing pulmonary edema. Pathological comparisons of lung samples obtained at initial diagnosis and at the time of transplantation, before and after the occurrence of $\mathrm{PH}$, provide possible explanations for the mechanisms of PH and the effectiveness of sildenafil in this patient.

\section{Case Report}

In November 2001, a 19-year-old man with a three-year history of smoking 20 cigarettes/day was diagnosed with

\footnotetext{
${ }^{1}$ First Department of Medicine, Hokkaido University School of Medicine, Japan, ${ }^{2}$ Department of Thoracic Surgery, Kyoto University, Japan, ${ }^{3}$ Faculty of Health Sciences, Hokkaido University, Japan, ${ }^{4}$ Department of Diagnostic Pathology, Kyoto University Hospital, Japan and ${ }^{5}$ Department of Cancer Pathology, Hokkaido University Graduate School of Medicine, Japan
} Received for publication September 16, 2013; Accepted for publication April 3, 2014 Correspondence to Dr. Satoshi Konno, satkonno@med.hokudai.ac.jp 




Figure 1. Clinical findings on high-resolution CT during follow-up visits. A: CT image at initial diagnosis (patient age 19 years). B: CT image seven years later. C: CT image upon admission to our hospital due to progressive PH. D: Postoperative CT image after right lung transplant



Figure 2. Overview of clinical, functional and hemodynamic features. CI: cardiac index, DLco: transfer factor for carbon monoxide, $\mathrm{FEV}_{1}$ : forced expiratory volume in $1 \mathrm{~s}$, ND: not determined, $\mathrm{PaO}_{2}$ : arterial oxygen tension, PAP: pulmonary arterial pressure, PFT: pulmonary function test, PVR: pulmonary vascular resistance, RHC: right heart catheterization, VC: vital capacity, WHO FC: WHO functional class. ${ }^{\#}$ At rest in room air.

PLCH from a biopsy of lung parenchymal and endobronchial lesions at a community hospital (Fig. 1A). The endobronchial lesions improved three months after he stopped smoking, but the lung parenchymal lesions remained unchanged. This particular clinical course has been reported previously (10). Three months after the initial diagnosis, the patient stopped attending the hospital and resumed smoking
(30 cigarettes/day) at 21 years of age.

At 26 years of age (May 2008), the patient attended the same hospital with progressive exertional dyspnea, which he had experienced for the past year and thereafter he was referred to our hospital for evaluation. Chest computed tomography (CT) revealed increased diffuse reticulonodular and cystic shadows when compared with the findings in 

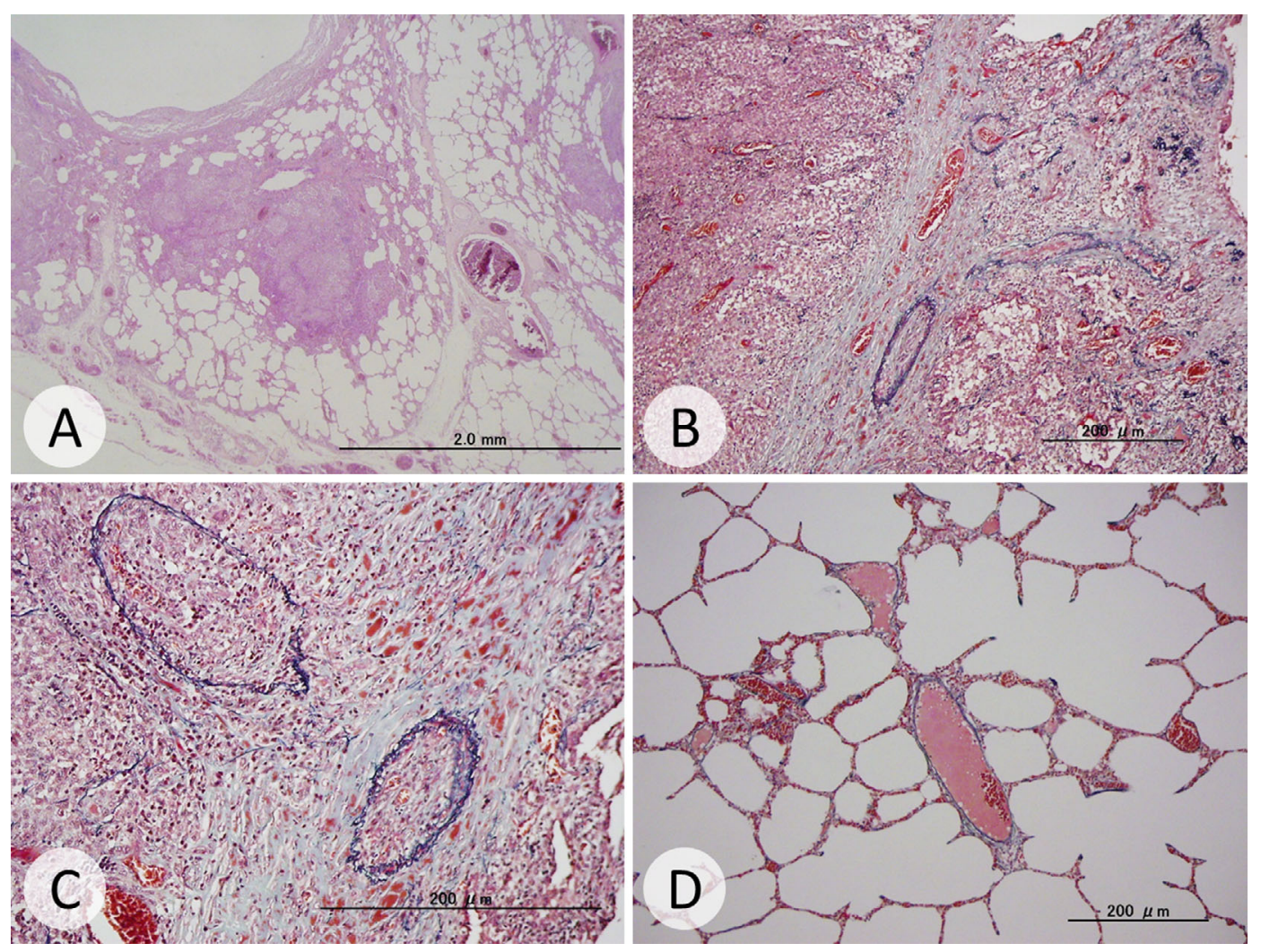

Figure 3. Pathological findings of lung biopsy obtained by VATS in absence of clinical signs of PH. A: Parenchymal multiple cystic lesions surrounded by nodular or asteroid granulomatous lesions involving eosinophils and histiocytic cells (Hematoxylin and Eosin staining, $\times 2$ ). B: Numerous inflammatory cells have migrated to interlobular septae including small arteries, arterioles, small vessels and venules (Elastica-Masson stain, $\times 10$ ). C: Several vessels are occluded or destroyed by inflammatory cell invasion and fibrosis (Elastica-Masson stain, $\times 20$ ). D: Minimal pulmonary vascular involvement in regions far from the parenchymal granulomatous lesions (Elastica- Masson stain, $\times 10$ )

2001 (Fig. 1B). The pulmonary function tests revealed reduced vital capacity (VC: $44.3 \%, 1.79 \mathrm{~L}$ ) and carbon monoxide diffusion capacity ( $\mathrm{DL}_{\mathrm{co}} \%$ predicted: $22.5 \%$ ) compared with the results at initial diagnosis (VC: $58.6 \%, \mathrm{DL}_{\mathrm{co}}$ \%predicted: $45.1 \%$; Fig. 2). Right heart catheterization (RHC) showed that the mean pulmonary arterial pressure (MPAP) had increased to $39 \mathrm{mmHg}$, and the pulmonary vascular resistance (PVR) was 587 dyn $\cdot \mathrm{s} \cdot \mathrm{cm}^{-5}$. Other RHC values were as follows: pulmonary capillary wedge pressure (PCWP) $4 \mathrm{mmHg}$, right ventricular end-diastolic pressure (RVEDP) $5 \mathrm{mmHg}$, cardiac output (CO)/cardiac index (CI) $4.8 \mathrm{~L} / \mathrm{min} / 3.1 \mathrm{~L} / \mathrm{min} / \mathrm{m}^{2}$, right arterial pressure (RAP) 5 $\mathrm{mmHg}$, and mixed venous oxygen saturation $\left(\mathrm{SvO}_{2}\right) 65 \%$. The overall clinical findings suggested progressive $\mathrm{PH}$ along with worsened parenchymal destruction. The patient then stopped smoking and began treatment with diuretics and long-term oxygen therapy (LTOT).

Despite treatment, the patient's dyspnea and hypoxia gradually worsened from the World Health Organization functional class (WHO-FC) II to IV, and he was admitted to our hospital in January 2010. Echocardiography showed severe $\mathrm{PH}$, whereas the lung parenchymal abnormalities on CT images were similar to those taken in 2008 (Fig. 1C), thus suggesting that the dyspnea and severe hypoxia were due to $\mathrm{PH}$ progression rather than worsening lung parenchymal lesions. Because treatment with prednisone $(60 \mathrm{mg} /$ day $)$ did not improve the dyspnea or hypoxia, the dose was rapidly tapered. The RHC at 50 days after resuming prednisolone $(20 \mathrm{mg} /$ day $)$ revealed advanced $\mathrm{PH}$ with increased MPAP $(60 \mathrm{mmHg})$ and PVR (628 dyn.s.cm $\left.{ }^{-5}\right)$.

Because of the sustained hypoxia and the absence of a clinical response to corticosteroids, the patient was put on sildenafil (60 mg t.i.d.) treatment in March 2010. At the 12month follow-up visit, significant improvements were evident in the WHO-FC (IV to III), MPAP (60 to $33 \mathrm{mmHg}$ ) and PVR (628 to 352 dyn $\cdot \mathrm{s} \cdot \mathrm{cm}^{-5}$ ) without any remarkable change in oxygenation [partial pressure of arterial oxygen $\left(\mathrm{PaO}_{2}\right)$ in room air: 47 to $\left.53 \mathrm{mmHg}\right]$.

The patient underwent a right single-lung transplant at Kyoto University in November 2011, which was 15 months after being placed on the transplant list (Fig. 1D). His postoperative hospital course was uneventful and sildenafil was discontinued. At the follow-up visit six months later, significant improvements were evident in the patient's WHO-FC (III to I), MPAP (15 mmHg), PVR (116 dyn $\left.\cdot \mathrm{s} \cdot \mathrm{cm}^{-5}\right)$ and oxygenation $\left(\mathrm{PaO}_{2}\right.$ in room air: 53 to $\left.76 \mathrm{mmHg}\right)$. The patient remained in good health at 18 months after lung transplantation without any clinical evidence of PLCH recurrence 

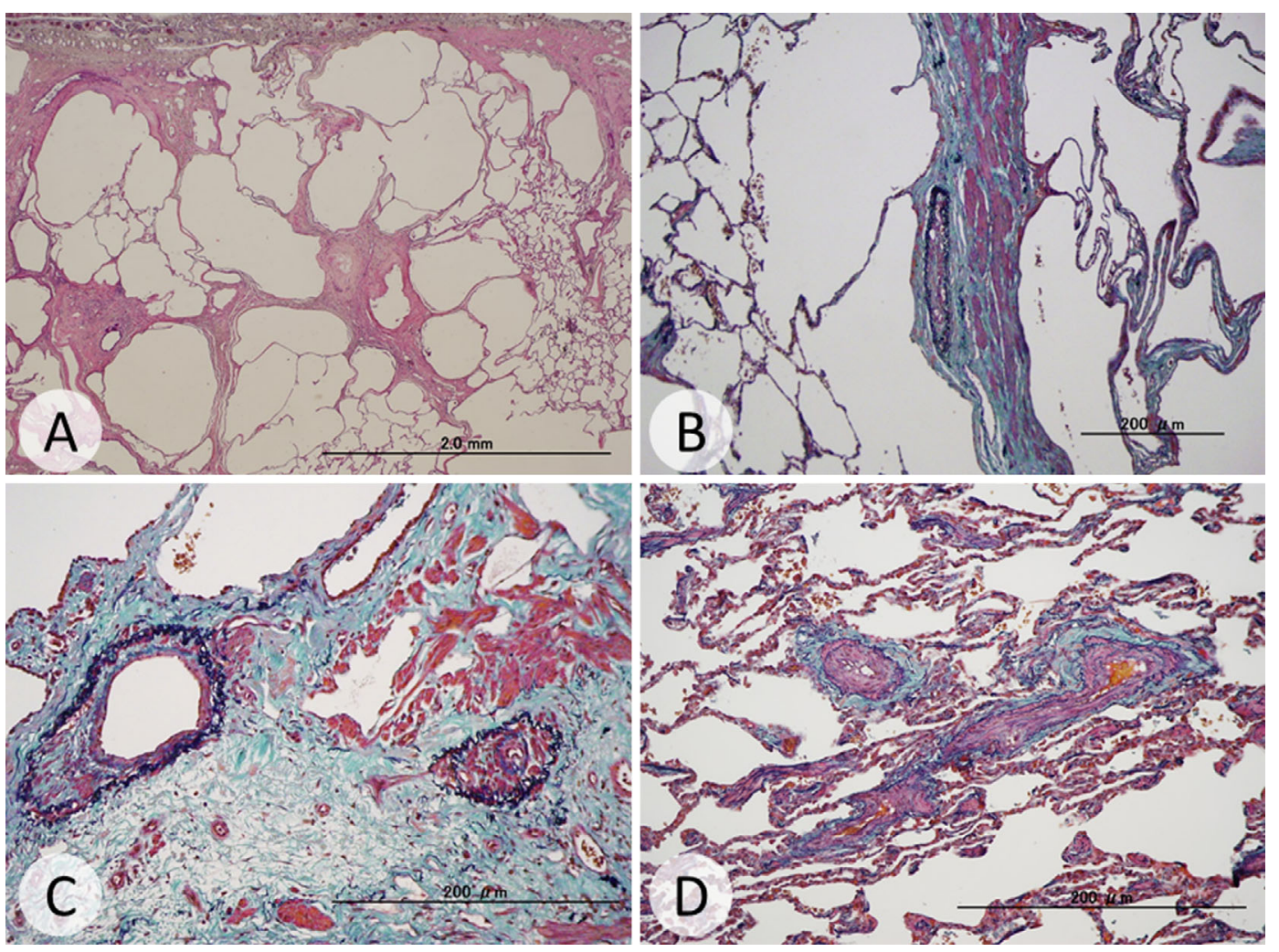

Figure 4. Pathological findings of the resected lung after right lung transplantation. A: Destruction of the parenchymal architecture by interstitial fibrosis and bronchocentric fibrous remodeling correspond to the advanced stage of PLCH (Hematoxylin and Eosin staining, $\times 2$ ). B: Massive fibrosis and smooth muscle cell proliferation in the interlobular septa including several vessels (ElasticaMasson stain, $\times 10$ ). C: Pulmonary vessels occluded by fibrosis with muscularization (Elastica-Masson stain, $\times 20$ ). D: Intimal thickening and medial hypertrophy of the pulmonary arteries/arterioles in the area of the preserved lung without obvious parenchymal fibrotic changes (Elastica-Masson stain, $\times 10$ ).

or a need for externally delivered oxygen.

\section{Pathological findings of lung samples obtained at the initial diagnosis and at lung transplantation}

The pathological findings before and during established $\mathrm{PH}$ were compared in the lung specimens obtained at the initial diagnosis and at transplantation. The pathological findings at the initial diagnosis included multiple parenchymal cystic lesions surrounded by nodular or asteroid granulomatous lesions (Fig. 3A). Many inflammatory cells consisted of CD1a-positive histiocytes, eosinophils and lymphocytes that had diffusely migrated from the lobules to the interlobular septae, including the small arteries, arterioles, small vessels and venules (Fig. 3B). Several vessels were occluded or destroyed by invasion of these cells and by fibrosis (Fig. 3C). However, the pulmonary vascular involvement was limited to the lung parenchyma far from the main granulomatous lesion (Fig. 3D).

Pathological findings in the resected right lung revealed that the parenchymal architecture had been destroyed by interstitial fibrosis and bronchocentric fibrous remodeling, but LCH cells were undetectable (Fig. 4A). The pulmonary architecture was destroyed (Fig. 4A), and massive fibrosis and smooth muscle cell proliferation were evident in the interlobular septae and in several vessels within the fibrotic lesions (Fig. 4B, C). The intimal thickening and medial hypertrophy that are features of PAH were apparent in some of the pulmonary arteries/arterioles in regions of the preserved lung without obvious parenchymal fibrotic changes (Fig. 4D). The capillary or lymphatic vessel dilation and intra-alveolar hemorrhage, which are characteristic of venoocclusive disease, were not detected in either of the lung samples. Immunohistochemical assessment revealed PDE5 staining on the pulmonary arterial/arteriolar walls in parenchymal fibrotic (Fig. 5A) and non-fibrotic lesions (Fig. 5B). The walls of the diseased pulmonary arteries/arterioles were also positive for endothelin receptor (ER) B staining (Fig. 5C), but not for ER-A (Fig. 5D).

\section{Discussion}

The pathophysiology of $\mathrm{PH}$ arising in PLCH, classified as group 5 according to the Dana Point classification system (11), is likely to be multifactorial. Chest CT of our patient showed advanced lung parenchymal destruction caused by cystic lesions. In addition, the patient had severe hypoxia 


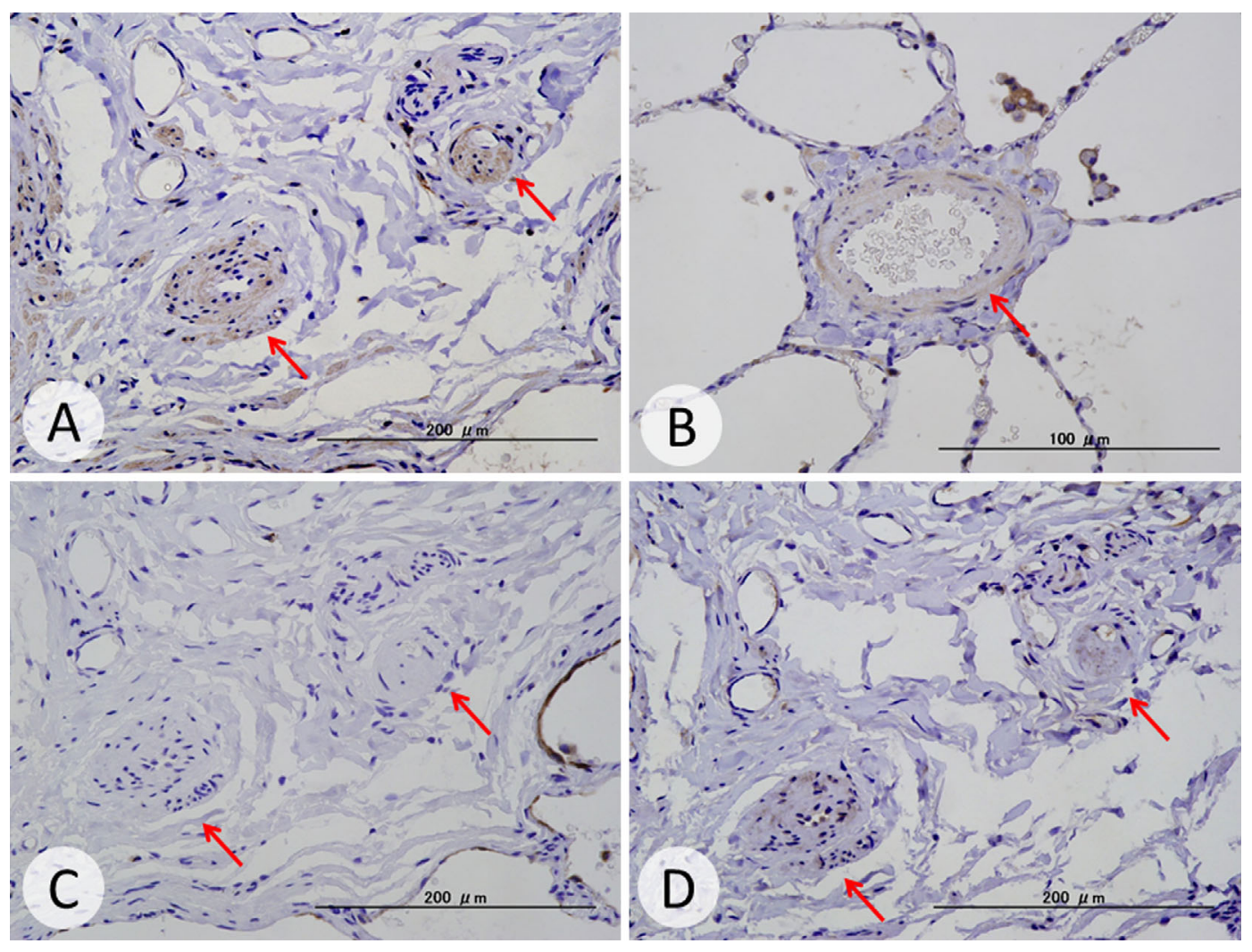

Figure 5. Immunohistochemical findings from lung tissues. Immunohistochemical staining of the resected right lung for phophodiesterase-5 (A: lung parenchymal fibrotic lesion, B: non-fibrotic lesion), endothelin receptor A (C) and endothelin receptor B (D). Arrows indicate diseased pulmonary arteries.

before starting LTOT. Therefore, the reduced pulmonary vascular bed caused by the cystic lesions and sustained hypoxia might have contributed to the development of $\mathrm{PH}$. Furthermore, the lung pathology at the initial diagnosis in the absence of PH-related clinical findings revealed fibrotic vascular abnormalities and vessel infiltration by $\mathrm{LCH}$ cells, with mild lung parenchymal destruction and cystic changes. Notably, the pathological findings in the resected right lung revealed vascular involvement in areas of the preserved lung without obvious parenchymal fibrotic changes in addition to the lung parenchymal fibrotic lesions. A study of 20 patients with PH-PLCH found no relationship between the severity of $\mathrm{PH}$ and impairment as determined by pulmonary function tests (12). Furthermore, in pathologically assessed lung samples of PH-PLCH patients before and after the occurrence of $\mathrm{PH}$, the pulmonary vasculature was worsened whereas the parenchymal lesions remained relatively unchanged (13). These published results, together with the present pathological findings, suggest that $\mathrm{PH}$ can develop independently of lung parenchymal damage and that the degree of lung vasculature involvement significantly contributes to the severity of PH in PLCH. Although the precise mechanisms of the development of vascular involvement in the area of preserved lung remains unknown, various cytokines or growth factors produced by LCH cells, as well as direct LCH cell invasion of vessels or sustained hypoxemia, may be related to pulmonary arterial remodeling (14-16).

The use of PAH-specific vasodilators to treat $\mathrm{PH}$ associated with chronic respiratory disease is considered to be hazardous because of the potential for a deterioration of a ventilation-perfusion mismatch and hypoxemia (17). However, recent studies have found that oxygenation does not worsen in patients with $\mathrm{PH}$ associated with chronic respiratory disease receiving PAH therapy (19). These findings are attributed to the disproportionately advanced or disproportionate $\mathrm{PH}$, characterized by developments that mechanical lung disturbances cannot sufficiently explain. Pavec et al. recently administered PAH therapy to 27 patients with $\mathrm{PH}-$ PLCH and found that oxygenation does not cause any deterioration (9). Sildenafil significantly improved the pulmonary hemodynamics in our patient without worsening hypoxemia. Vasodilators can theoretically dilate vessels in poorly ventilated lung fields, which can exacerbate a ventilation-perfusion mismatch and hypoxia. Indeed, sildenafil may have similarly affected pulmonary gas oxygenation in the fibrotic area of our patient. However, lung specimens obtained from our patient at transplantation showed intimal thickening and medial hypertrophy in some pulmonary arteries in areas with preserved alveolar architecture that were distant from the parenchymal PLCH lesions. Immunohistochemical staining revealed PDE5 expression in the diseased pulmonary artery in the area of the preserved lung. 
Therefore, we speculate that sildenafil dilated the pulmonary arteries predominantly in lesions that were located far from parenchymal PLCH in our patient, thereby minimizing the ventilation-perfusion mismatch. The fact that sildenafil enhances the nitric oxide pathway in well-ventilated lung areas (20) supports this speculation.

Although the additional risk for pulmonary edema caused by vasodilators in patients with PH-PLCH has been discussed $(13,18)$, our patient did not develop pulmonary edema after sildenafil treatment. In addition, capillary dilation and intra-alveolar hemorrhage or lymphangiectasis, which are characteristic of veno-occlusive disease (13), were scarce. Furthermore, venous involvement was modest in the fibrotic area and not widely distributed in areas of the preserved lung. Thus, the vasculopathy that predominated in the arteries/arterioles might explain why sildenafil did not cause pulmonary edema in our patient.

In the present case, immunohistochemical staining of PDE5 and ER-B was positive in the diseased pulmonary arteries/arterioles. We also previously described the effectiveness of using a PDE5 inhibitor and an ER blockade to treat PH combined with pulmonary fibrosis and emphysema (CPFE) which showed positive staining of PDE5 and ER-B in diseased pulmonary vessels (21). Further studies with more patients are necessary to clarify the association between the positive PDE5/ER seen in the immunohistochemical analysis and the clinical response to these two PAHspecific vasodilators.

In summary, we herein described PH-PLCH in a patient in whom sildenafil significantly improved his pulmonary hemodynamics without causing any worsening of oxygenation or pulmonary edema. The pathological findings at lung transplantation provided possible explanations for these significant effects. Together with the report published by Pavec et al., the present findings suggest that sildenafil could be a therapeutic option for PH-PLCH. However, further studies are warranted to evaluate the safety and effectiveness of $\mathrm{PAH}$-specific vasodilators in patients with PH-PLCH.

The authors state that they have no Conflict of Interest (COI).

\section{References}

1. Abbott GF, Rosado-de-Christenson ML, Franks TJ, Frazier AA, Galvin JR. From the archives of the AFIP: pulmonary Langerhans cell histiocytosis. Radiographics 24: 821-884, 2004.

2. Sundar KM, Gosselin MV, Chung HL, Cahill BC. Pulmonary Langerhans cell histiocytosis: emerging concepts in pathobiology, radiology, and clinical evolution of disease. Chest 123: 16731683,2003

3. Tazi A. Adult pulmonary Langerhans' cell histiocytosis. Eur Respir J 27: 1272-1285, 2006.
4. Vassallo R, Ryu JH, Colby TV, Hartman T, Limper AH. Pulmonary Langerhans'-cell histiocytosis. N Engl J Med 342: 19691978, 2000.

5. Basset F, Corrin B, Spencer H, et al. Pulmonary histiocytosis X. Am Rev Respir Dis 118: 811-820, 1978.

6. Delobbe A, Durieu J, Duhamel A, Wallaert B. Determinants of survival in pulmonary Langerhans' cell granulomatosis (histiocytosis X). Groupe d'Etude en Pathologie Interstitielle de la Societe de Pathologie Thoracique du Nord. Eur Respir J 9: 2002-2006, 1996.

7. Vassallo R, Ryu JH, Schroeder DR, Decker PA, Limper AH. Clinical outcomes of pulmonary Langerhans'-cell histiocytosis in adults. N Engl J Med 346: 484-490, 2002.

8. Kiakouama L, Cottin V, Etienne-Mastroïanni B, Khouatra C, Humbert M, Cordier JF. Severe pulmonary hypertension in histiocytosis X: long-term improvement with bosentan. Eur Respir J 36: 202-204, 2010.

9. Le Pavec J, Lorillon G, Jaïs X, et al. Pulmonary Langerhans Cell Histiocytosis associated pulmonary hypertension: clinical characteristics and impact of pulmonary arterial hypertension therapies. Chest 142: 1150-1157, 2012.

10. Suzuki M, Betsuyaku T, Suga M, Ishizu A, Nishimura M, Oguri M. Pulmonary Langerhans' cell histiocytosis presenting with an endobronchial lesion. Intern Med 43: 227-230, 2004.

11. Galiè N, Hoeper MM, Humbert M, et al; ESC Committee for Practice Guidelines (CPG). Guidelines for the diagnosis and treatment of pulmonary hypertension: the Task Force for the Diagnosis and Treatment of Pulmonary Hypertension of the European Society of Cardiology (ESC) and the European Respiratory Society (ERS), endorsed by the International Society of Heart and Lung Transplantation (ISHLT). Eur Heart J 30: 2493-2537, 2009.

12. Harari S, Comel A. Pulmonary Langerhans cell Histiocytosis. Sarcoidosis Vasc Diffuse Lung Dis 18: 253-262, 2001.

13. Fartoukh M, Humbert M, Capron F, et al. Severe pulmonary hypertension in histiocytosis X. Am J Respir Crit Care Med 161: 216-223, 2000.

14. Tazi A, Bonay M, Grandsaigne M, Battesti JP, Hance AJ, Soler P. Surface phenotype of Langerhans' cells and lymphocytes in granulomatous lesions from patients with pulmonary histiocytosis X. Am Rev Respir Dis 147: 1531-1536, 1993.

15. Asakura S, Colby TV, Limper AH. Tissue localization of transforming growth factor-beta 1 in pulmonary eosinophilic granuloma. Am J Respir Crit Care Med 154: 1525-1530, 1996.

16. Sauder DN, Dinarello CA, Morhenn VB. Langerhans' cell production of interleukin-1. J Invest Dermatol 82: 605-607, 1984.

17. Simonneau G, Escourrou P, Duroux P, Lockhart A. Inhibition of hypoxic pulmonary vasoconstriction by nifedipine. $\mathrm{N}$ Engl J Med 304: 1582-1585, 1981.

18. Hamada K, Teramoto S, Narita N, Yamada E, Teramoto K, Kobzik L. Pulmonary veno-occlusive disease in pulmonary Langerhans' cell granulomatosis. Eur Respir J 15: 421-423, 2000.

19. Igarashia A, Sato T, Tsujino I, et al. Four cases with group 3 outof-proportion pulmonary hypertension with a favorable response to vasodilators. Respir Med Case Reports 9: 4-7, 2013.

20. Galie N, Ghofrani HA, Torbicki A, Barst RJ, Rubin LJ, Badesch D. Sildenafil citrate therapy for pulmonary arterial hypertension. N Engl J Med 353: 2148-2157, 2005.

21. Sato T, Tsujino I, Tanino M, Ohira H, Nishimura M. Broad and heterogeneous vasculopathy in pulmonary fibrosis and emphysema with pulmonary hypertension. Respirology Case Reports 1: 10-13, 2013.

(C) 2014 The Japanese Society of Internal Medicine http://www.naika.or.jp/imonline/index.html 